\title{
Enfoque del paciente con cefalea en tiempos de covid-19
}

\author{
Approach to the patient with headache in times of COVID-19
}

\section{Carolina Ospina (1), Michel Volcy (2)}

\begin{abstract}
RESUMEN
Ante el numero cada vez mayor de pacientes con covid-19 y los rápidos cambios que ha traido consigo la pandemia en los estilos de vida, se redactó el presente documento, con el objetivo de proveer una recomendación a los neurológos y médicos en general para el abordaje del paciente con cefalea. Inicialmente, se realiza el abordaje de la cefalea como uno de los síntomas del covid-19, revisando las series de casos y proponiendo posibles clasificaciones diagnósticas para explicar su presencia en estos pacientes. Posteriormente, se evaluará la cefalea secundaria a otros trastornos neurológicos tambien reportados en pacientes con covid-19 y los signos de alarma para realizar estudios complementarios. Por último se revisará como ha cambiado el seguimiento de los pacientes con cefalea primaria en medio de la pandemia, recomendaciones sobre ajustes que se deben hacer durante este tiempo en su manejo y formulación, y finalmente recomendaciones sobre interacciones en sus medicamentos en caso de enfermar por covid-19.
\end{abstract}

PALABRAS CLAVE: aislamiento, cefalea, covid-19, Migraña (DeCS).

\section{SUMMARY}

Given the increasing number of patients with COVID-19 and the rapid changes that the pandemic has brought about in lifestyles, this document was developed with the aim of providing a recommendation to neurologists and doctors in general for the approach of the patient with headache. Initially, the headache was treated as one of the symptoms of COVID-19, reviewing the case series and proposing possible diagnostic classifications to explain its presence in these patients. Subsequently, headache secondary to other neurological disorders also reported in patients with COVID-19 and the warning signs will be evaluated to carry out complementary studies. Finally, it will be reviewed how the follow-up of patients with primary headache in the midst of the pandemic has changed, recommendations on adjustments to be made during this time in its management and formulation, and finally recommendations on drug interactions in case of illness due to COVID-19.

KEYWORDS: Migraine disorders, headache, patient isolation, coronavirus (MeSH).

\section{INTRODUCCIÓN}

Con base en estudios previos y la experiencia con otros virus de la familia coronavirinae, se ha propuesto que el covid-19, además del compromiso respiratorio, tiene potencial de neuroinvasión (1). Lo que inicialmente eran hipotesis, la literatura científica más reciente tiende a confirmarlo. El pasado mes de abril se publicó un reporte de caso de un paciente post mortem con presencia de covid-19 en células neurales y endoteliales del lóbulo frontal (2). Se logró asimismo la identificación del virus por técnica de RT-PCR en el líquido cefalorraquídeo de un paciente con meningoencefalitis (3). De allí la importancia de un papel activo del neurólogo en la pandemia.

\section{CEFALEA COMO SÍNTOMA DE LA INFECCIÓN POR COVID-19}

En cuanto a la cefalea, se calcula que la frecuencia en pacientes con covid-19 puede variar entre $6,5 \%$ y $34 \%$

(1) Residente de cuarto año de Neurología en la Universidad de Antioquia, médica egresada de esa misma institución

(2) Neurólogo de la Universidad de Antioquia, especialista en cefaleas. Director del Instituto de Dolor de Cabeza y Enfermedades Neurológicas (Indocen), profesor de Neurología de la Universidad de Antioquia.

Recibido 27/5/20. Aceptado:5/6/20. 
(4-7) y en muchos casos hace parte de los síntomas iniciales de la infección (7-10). Es necesario aclarar que estos datos epidemiológicos provienen de pacientes que fueron atendidos en centros hospitalarios y no existe hasta el momento información suficiente sobre la frecuencia en pacientes con síntomas leves que no requirieron acudir a instituciones de salud (tabla 1). Puesto que los pacientes consultan al servicio de urgencias por cefaleas y que esto corresponde hasta el 10\% del total de consultas al día, es importante resaltar que pueden haber otras causas de cefalea que motiven la consulta en urgencias (11).

Hasta este punto, la cefalea haría parte del grupo de síntomas inespecíficos, que pueden presentarse durante la infeccion y no necesariamente reflejar la presencia de una complicación neurológica. Otros de estos síntomas frecuentes de la infección por covid-19 son fiebre, fatiga, tos, nauseas y vómito (13). Aunque la frecuencia de presentacion de los síntomas varía de acuerdo con el estudio, Mao y colaboradores analizaron la frecuencia de síntomas generales de la infección y de síntomas neurológicos asociados en pacientes hospitalizados (6) (tabla 2).

En dicho contexto, la cefalea como síntoma inicial podría orientarse a una cefalea secundaria a infección. De acuerdo con la clasificación ICHD3, la triada de cefalea, fiebre y nauseas o vómito es altamente sugestiva de "cefalea atribuida a infección" (tabla 3) (14). Adicionalmente, de acuerdo con el potencial de compromiso de otros órganos, pudiera estarse en presencia de: cefalea debido a infección viral sistémica ICHD3 9.2.2, cefalea primaria por tos ICHD3 4.1, cefalea asociada a hipoxia o a hipercapnia ICHD3 10.1, cefalea asociada a heteroforia ICHD3 A11.3.5 (15). Esto indica diversos mecanismos fisiopatológicos involucrados.

Pacientes con antecedentes de cefaleas primarias podrían presentar infección por covid-19. De acuerdo con la clasificación ICHD3, en este escenario se requiere categorizar cada uno de los diagnósticos con el subtipo o subforma de dolor. Por ejemplo:

- En pacientes sin historia de cefalea: cuando ocurre una cefalea por primera vez en relación temporal con una infección, se codifica como un dolor de cabeza secundario atribuido a esa infección, incluso si tiene las características sugestivas de cefalea primaria (14). El diagnostico sería: cefalea debida a infección viral sistemica (ICHD3 9.2.2).

- En pacientes con antecedentes de cefalea primaria: si se cronifica o empeora en intensidad o frecuencia, en estrecha relación temporal con una infección, deben darse ambos diagnósticos, cefalea atribuida a infección y con el diagnóstico de cefalea previa (14). Es decir, cefalea debida a infección viral sistemica (ICHD3 9.2.2) y migraña con aura (ICHD3 migraña con aura 1.2).

Las cefaleas primarias son un trastorno frecuente en la población general. Se calcula que la migraña afecta a cerca del $15 \%$ de los adultos alrededor del mundo, y la cefalea tipo tensional a un $26 \%$ (15).

Por ello, no es improbable que dentro del grupo de pacientes infectados con coronavirus se tengan pacientes con antecedentes de cefaleas primarias. Pozo y colaboradores, en el "Resident's initiative to study headache during coronavirus epidemic: the RESIST-HeaDaCHE study",

Tabla 1. Frecuencia de la cefalea en urgencias, comparativo Covid-19 vs. no Covid-19

\begin{tabular}{|c|c|c|c|}
\hline \multirow{2}{*}{$\begin{array}{l}\text { Cefalea en pacientes por Covid-19 } \\
\text { Cefalea como síntoma inicial (12) }\end{array}$} & \multicolumn{3}{|c|}{ Cefalea en pacientes no Covid por urgencias (11) } \\
\hline & $6 \%-10 \%$ & Cefaleas primarias & $11 \%$ \\
\hline Cefalea en pacientes hospitalizados & $6,5 \%-34 \%$ & Cefalea por infección viral sistémica & $22 \%$ \\
\hline \multirow[t]{9}{*}{ Cefalea en pacientes que requieren manejo en UCI (4-7) } & $6,5 \%-17 \%$ & Cefalea por infección respiratoria alta & $16 \%$ \\
\hline & & Cefalea por enfermedad diarreica & $8 \%$ \\
\hline & & Cefalea por hipertensión arterial & $5 \%$ \\
\hline & & Cefalea por fiebre & $5 \%$ \\
\hline & & Cefalea asociada a enfermedad coronaria & $4 \%$ \\
\hline & & Cefalea por aneurisma carotideo & $1 \%$ \\
\hline & & Cefalea por trauma & $1 \%$ \\
\hline & & Cefalea por meningitis & $1 \%$ \\
\hline & & Otras cefaleas secundarias & $12 \%$ \\
\hline
\end{tabular}

Fuente: tomado y modificado de $(4-7,11,12)$. 
describen en resultados preliminares la presencia de $32 \%$ de pacientes con antecedente de migraña.

En este momento no hay muchos estudios sobre las características semiológicas de la cefalea en covid-19 que permitan diferenciarla de otras cefaleas primarias. En general, se le describe como de intensidad moderada, opresiva o pulsátil, con una localización temporoparietal o hacia la región anterior o en el área periorbital, que suele iniciar al mismo tiempo que los otros síntomas de covid-19 como la fiebre, las mialgias, la fatiga, la anosmia y la ageusia, y que se limita a la fase activa de la enfermedad (12). Aunque la literatura aún no lo describe, por carecer de análisis de seguimiento longitudinal, podría ser parte del desarrollo de una cefalea persistente crónica de novo secundaria.

Tabla 2. Síntomas frecuentes en infección por Covid-19

\begin{tabular}{|c|c|c|c|}
\hline \multicolumn{4}{|c|}{ Síntomas de Covid-19 } \\
\hline \multicolumn{2}{|c|}{ Síntomas generales } & \multicolumn{2}{|c|}{ Síntomas neurológicos } \\
\hline Fiebre & $62 \%$ & Mareo & $17 \%$ \\
\hline Tos & $50 \%$ & Cefalea & $13 \%$ \\
\hline Anorexia & $32 \%$ & $\begin{array}{l}\text { Alteración de } \\
\text { la consciencia }\end{array}$ & $8 \%$ \\
\hline Diarrea & $19 \%$ & Disgeusia & $6 \%$ \\
\hline Odinofagia & $15 \%$ & Hiposmia & $5 \%$ \\
\hline
\end{tabular}

Fuente: tomado y modificado de Mao y colaboradores (6)
Por todos estos motivos, es importante recordar que el diagnóstico de cefalea se basa en un adecuado interrogatorio y un examen físico neurológico (tabla 4).

Debe considerarse que tratamientos empíricos de covid19 como hidroxicloroquina, cloroquina, lopinavir/ritonavir y tocilizumab pueden generar cefalea como evento adverso compatible con ICHD3, cefalea atribuida al uso ocasional de medicamentos no usados para la cefalea 8.1.9 (18).

\section{Cefalea como síntoma de otros trastornos neurológicos en covid-19}

Desde el punto de vista de neuroinfección, en la ICHD3, de acuerdo con dos reportes de caso en covid-19 con meningo-encefalitis y uno de encefalopatía necrotizante aguda hemorrágica, la cefalea presente en estos pacientes se categorizaría como cefalea atribuida a meningitis o encefalitis viral, de acuerdo con la ICHD3 (tabla 5).

Todos estos conceptos hacen necesario considerar la pertinencia de estudios paraclínicos en esta población de pacientes, en especial en sujetos con examen neurológico anormal, banderas rojas, cefalea tipo cluster primer diagnóstico, entre otros (tabla 6). Igualmente, se debe resaltar por medio de diferentes análisis que las recomendaciones basadas en la evidencia, la realización de neuroimágenes, resonancia magnética cerebral y tomografía de cráneo es $\mathrm{C}$, lleva a la conclusión de que en pacientes con covid-19 (19) la recomendación inicial es TAC de cráneo.

Tabla 3. Criterios diagnósticos de cefalea debida a infección viral sistémica

\section{Cefalea debida a infección viral sistémica}

A. Cefalea de cualquier duración que cumple el criterio $\mathrm{C}$

B. Ambos criterios

1. Infección viral sistémica diagnosticada

2. No hay evidencia de compromiso meníngeo o de encefalitis

C. Evidencia de causalidad demostrada por al menos dos de los siguientes criterios:

1. Cefalea que se ha desarrollado en relación temporal con el inicio de la infección viral sistémica

2. Cefalea que ha empeorado significativamente de forma paralela a la progresión de los síntomas de la infección viral sistémica

3. El dolor de cabeza mejoró o se resolvió significativamente en paralelo con la mejoría o la resolución de la infección viral sistémica

4. Cefalea que tiene una o ambas características:

a) Dolor difuso

b) Intensidad moderada o severa

D. No se explica mejor por otro diagnóstico de ICHD3

Fuente: tomado y modificado de ICHD3 (14). 
Tabla 4. Valor de variables clínicas en potencial de predicción en diagnóstico de la migraña

Lipton y colaboradores (16)

Detsky y colaboradores (17)

\begin{tabular}{|c|c|c|c|c|}
\hline Característica clínica & Sensibilidad & Especificidad & VPP & Con la mnemotecnia Pounding \\
\hline Nauseas & $60 \%$ & $81 \%$ & & Cuatro criterios LR de migraña definitiva o posible: 24 \\
\hline Fotofobia & $75 \%$ & $74 \%$ & & Tres criterios LR: 3.5 \\
\hline Fonofobia & $83 \%$ & $56 \%$ & & Dos o menos LR: 0.41 \\
\hline Aura & $43 \%$ & $74 \%$ & & \\
\hline Limitación funcional & $87 \%$ & $52 \%$ & & \\
\hline $\begin{array}{l}\text { Nausea }+ \text { fotofobia } \\
+ \text { limitación funcional }\end{array}$ & $81 \%$ & $75 \%$ & $93 \%$ & \\
\hline \multicolumn{5}{|c|}{$\begin{array}{l}\text { Abreviaturas: VPP: valor predictivo positivo. LR: likelihood ratio o razón de verosimilitud. pounding mnemotecnia: pulsación, } \\
\text { duración de 4-72 horas, unilateral, náuseas, incapacitante. }\end{array}$} \\
\hline
\end{tabular}

Tabla 5. Criterios diagnósticos de cefalea atribuida a meningitis o encefalitis viral.

\section{Cefalea atribuida a meningitis o encefalitis viral}

A. Cefalea de cualquier duración que cumple el criterio C

B. Diagnóstico de meningitis o encefalitis viral

C. Evidencia de causalidad demostrada por al menos dos de los siguientes criterios:

1. Cefalea que se desarrolla en relación temporal con el inicio de la meningitis o encefalitis viral

2. Cefalea que ha empeorado significativamente de forma paralela a la progresión de los síntomas de la infección viral sistémica

3. El dolor de cabeza mejoró o se resolvió significativamente en paralelo con la mejoría o la resolución de la infección viral sistémica.

4. Cefalea que tiene al menos una de las siguientes características:

a) Holocraneal

b) Localizada en la región del cuello y rigidez de cuello

D. No se explica mejor por otro diagnóstico de ICHD3

Fuente: tomado y modificado de ICHD3 (14).

\section{CEFALEA EN EL PERSONAL DE SALUD}

La cefalea no es un síntoma exclusivo de los pacientes con covid-19. El estudio HAPPE encontró que en el personal de salud el $81 \%$ desarrolló cefalea de novo asociada al equipo de protección personal, y hasta 93,5\% entre los que tenían antecedente de cefalea primaria. También fue más frecuente en aquellas personas con más de cuatro horas de uso al día del equipo de protección personal. Según este estudio, en la mayoría de los pacientes la localización del dolor era bilateral, opresivo, con mayor intensidad en los puntos de presión. De la misma manera, la latencia para el inicio de los síntomas era menor a una hora en el 81,3\% de los casos, y después de retirar el equipo de protección personal se resolvía por sí misma en menos de una hora en el 95,3\% (20), compatible con cefalea por presión externa ICHD3 4.6.1 (14).

En estos casos se recomienda evitar el uso de opioides en el manejo del dolor, asegurarse de que el equipo de protección se ajuste y posicione adecuadamente, y propender por una rotación de turnos que permita el descanso adecuado en el personal de salud. En el caso de profesionales con antecedente de cefalea primaria, es preciso garantizar la continuidad de su manejo preventivo (21). 


\section{Tabla 6. Indicaciones para neuroimagen en cefaleas primarias}

Indicación de neuroimagen

LR para observar una anormalidad intracraneal seria en la neuroimagen

\begin{tabular}{lll}
\hline & LR+ & LR- \\
\hline Cefalea con características de cefalea tipo cluster & 11 & 0,95 \\
\hline Examen neurológico anormal & 5,3 & 0,71 \\
\hline Cefalea mal definida & 3,8 & 0,66 \\
\hline Cefalea con aura & 3,2 & 0,51 \\
\hline Cefalea con síntomas focales & 3,1 & 0,79 \\
\hline Cefalea desencadenada por valsalva o ejercicio & 2,3 & 0,7 \\
\hline Cefalea con vómito de novo & 1,8 & 0,47 \\
\hline
\end{tabular}

Abreviaturas LR: likelihood ratio o razón de verosimilitud.

Fuente: datos tomados y modificados de (17)

\section{SEGUIMIENTO DE LOS PACIENTES CON CEFALEA PRIMARIA EN MEDIO DE LA PANDEMIA}

Si bien no se ha documentado que los pacientes con cefalea primaria sean una población de riesgo para contraer covid-19, la situación actual de aislamiento, teletrabajo, entre otras características, sí los expone a un mayor riesgo de agudización del cuadro clínico en relación con la presencia de múltiples factores de riesgo modificables (22):

- Aumento en los niveles de estrés.

- Trastornos del ánimo.

- Trastornos del sueño.

- Condiciones laborales diferentes con exposición prolongada a pantallas por teletrabajo, incluyendo las labores diarias en el trabajo, reuniones virtuales, entre otros. El impacto en el dolor por migraña se explica por las diferentes vías de estimulación de la corteza visual y del tálamo a partir de las señales transmitidas desde el globo ocular y la retina; por ello es importante la protección de la exposición visual (23).

- Cambios de condiciones ambientales.

- Utilización de dispositivos como micrófonos, audífonos, diademas, máscaras faciales con amarre craneano, lo que agudizaría los síntomas en pacientes con alodinia.

- Cambios en los patrones y horarios de alimentación.

Ante la situación actual, la telemedicina es una alternativa que permite garantizar al paciente el acompañamiento, la prescripción de su manejo y la continuidad del tratamiento, reconociendo que el examen neurológico puede quedar en algunos aspectos incompleto, lo que requeriría la necesidad de atención presencial. Si se considera todo el tema del diag- nóstico de cefaleas primarias, se puede tener un diagnóstico presuntivo o definitivo con un valor predictivo positivo cercano al 93,3\%. Adicionalmente, se ha demostrado niveles de satisfacción similares a los de la consulta presencial (24), comodidad en los pacientes por tiempo y costos de desplazamiento (25), resultados similares a la consulta presencial en desenlaces como mejoría subjetiva de la cefalea, puntaje en el HIT-6 a los tres meses de seguimiento $(24,26)$, así como adherencia terapéutica (24).

En un estudio reciente, Grazzi y colaboradores describen el potencial beneficio de estrategias de acompañamiento y terapias comportamentales por telemedicina en pacientes con cefalea por sobreuso de medicamentos (27). Por todos estos motivos, las aseguradoras deben continuar garantizando el acceso de los pacientes a sus controles por neurología, sea de forma presencial o por telemedicina.

\section{TRATAMIENTO DE LOS PACIENTES CON CEFALEA PRIMARIA EN MEDIO DE LA PANDEMIA}

En pacientes con cefaleas primarias no se deben olvidar estos conceptos:

- El manejo agudo busca disminuir la duración y la intensidad de la cefalea o sus síntomas acompañantes. Por ello, se recomienda iniciar de manera temprana, idealmente dentro de las dos primeras horas a partir del inicio de la crisis (28).

- Se cuenta con medicamentos de uso ambulatorio específicos que incluyen los triptanes y los ergotamínicos (28). Sin embargo, estos medicamentos no deben usarse simultáneamente pues se potencia el efecto vasoespástico y con ello el riesgo de efectos secundarios (29). 
- En pacientes en manejo con zolmitriptan, debe educarse para no compartir los dispositivos de administración nasal.

- No se recomienda el uso de opioides para el manejo agudo pues aumentan el riesgo de cefalea por uso excesivo de analgésicos (28).

- En el caso de pacientes que presentan una crisis de dolor prolongada, debe ofrecerse una alternativa de manejo para aplicar ambulatoriamente antes de decidir consultar por el servicio de urgencias; en este caso se usarían las terapias de transición (30).

- El ácido valproico puede ser útil como medicamento preventivo o terapia de transición; sin embargo, debe vigilarse el riesgo de toxicidad hepática, en especial por la combinación de otros medicamentos de uso potencial en covid-19 con metabolismo hepático.

- Respecto a los antagonistas de receptores angiotensina II, como el candesartan en uso preventivo (28), se carece de información que haga necesaria la suspensión.

En la elección del manejo preventivo para pacientes con migraña lo más importante es que se garantice el acceso al medicamento y la continuidad de este, pero además deben considerarse los cambios en el estilo de vida como consecuencia del aislamiento que predisponen con mayor frecuencia a ciertas condiciones médicas; estas deben tenerse en cuenta al momento de elegir el manejo profiláctico por su perfil de riesgos y beneficios (tabla 7).

En personas que estuvieran en manejo con toxina botulínica o bloqueos pericraneales, y que hagan parte de la población de alto riesgo para covid-19, o que prefieran posponer la aplicación de esta por las medidas de aislamiento, deben evaluarse alternativas diferentes y ofrecerse terapias de transición (37). En caso de que no sea posible cambiar el manejo, no existen recomendaciones sobre las medidas de seguridad en la aplicación de estos medicamentos. Por ello, se deben adoptar las recomendaciones que se han hecho para otros procedimientos ambulatorios, que en Colombia están consignadas en el documento Orientaciones para la restauración gradual de los servicios de salud en las fases de mitigación y control de la emergencia sanitaria por covid-19 en Colombia, publicado el pasado 5 de mayo del 2020 (38).

El Ministerio de Salud, en cuanto a las recomendaciones para el uso del equipo de protección personal, en el documento Lineamientos para prevención control y reporte de accidente por exposición ocupacional al covid-19 en instituciones de salud (39) incluye las que se muestran a continuación. Sin embargo, no se aclara si tales recomendaciones son para protección ante pacientes covid-19 o pacientes en general:

- Trabajador de la salud en contacto directo con el paciente en procedimientos que no generan aerosoles: mascarilla quirúrgica, visor, careta o monogafas, bata manga larga antifluido, guantes no estériles, vestido quirúrgico debajo de la bata que se retira al final del turno. Opcional: gorro.

- Paciente: mascarilla quirúrgica de acuerdo con tolerancia.

Los anticuerpos monoclonales antagonistas del péptido relacionado con el gen de la calcitonina (CGRP) y el agonista selectivo del receptor de serotonina $1 \mathrm{~F}$ hacen parte del nuevo desarrollo de medicamentos profilácticos y abortivos, respectivamente. El antagonista intranasal del CGRP vazegepant estaría siendo evaluado en ensayo clínico de seguridad y eficacia para pacientes hospitalizados con covid-19, con la hipótesis de generar disminución de respuesta inflamatoria severa alveolar y el consiguiente retraso en el desarrollo de desaturación de oxígeno, SDRA, requerimiento de oxigenación suplementaria, ventilación artificial o muerte (40). Por lo nuevo y reciente del uso de estos medicamentos, la literatura aún no menciona los beneficios o riesgos en la población con o sin covid-19 (tabla 7).

Si se considera la ubicuidad del CGRP y de los receptores, el mecanismo de acción y las vías moleculares involucradas, es importante tener en cuenta el riesgo potencial de estos medicamentos, pues el CGRP desempeña varios papeles en la protección cardiovascular contra la hipertensión, la isquemia, la insuficiencia cardiaca y la adaptación vascular en el embarazo. El bloqueo a largo plazo de CGRP podría exacerbar una enfermedad cardiovascular previa o incrementar la severidad de nuevos eventos cardiovasculares $(41,42)$. Asimismo, el CGRP tiene un rol protector en el sistema nervioso central como buffer del calcio intracelular. De tal modo, activa cascadas de señalización antiapoptóticas, aumenta los niveles de las neurotrofinas, disminuye el edema cerebral y mantiene la barrera hematoencefálica intacta para limitar el paso de neutrófilos y monocitos (43).

\section{RECOMENDACIONES PARA EL MANEJO DE PACIENTES CON CEFALEA PRIMARIA E INFECCIÓN POR COVID-19}

Dentro del arsenal de medicamentos para el manejo agudo de la cefalea están el acetaminofén, la metoclopramida, la domperidona y los fármacos antiinflamatorios no esteroideos (AINES) (28). Si bien inicialmente se consideró que estos últimos podrían exacerbar los síntomas de covid-19, por su mecanismo de acción a través de la enzima convertidora de angiotensina (45), la Food and Drug Administration (FDA) a mediados de marzo expresó que no había evidencia científica que lo confirmara (46) y posteriormente la Organización Mundial de la Salud (OMS) publicó una revisión en la que se afirma que en la actualidad no hay evidencia de eventos adversos graves, impacto en la supervivencia a largo plazo o en la calidad de vida en pacientes con covid-19 como resultado del uso de AINES

Acta Neurol Colomb. 2020; 36(2): Supl 1.: 27-38. 
Tabla 7. Condiciones médicas que se deben considerar al momento de elegir el profiláctico

\begin{tabular}{|c|c|c|c|c|c|c|c|}
\hline & $\begin{array}{l}\text { Cambios } \\
\text { en el gasto } \\
\text { cardíaco }\end{array}$ & Depresión & $\begin{array}{l}\text { Obesidad/ } \\
\text { aumento de } \\
\text { peso }\end{array}$ & $\begin{array}{l}\text { Visión } \\
\text { borrosa/ } \\
\text { glaucoma }\end{array}$ & Dispepsia & Edemas & \begin{tabular}{|l} 
Insomnio/ \\
parasomnias
\end{tabular} \\
\hline Acido valpro & & & & & & & \\
\hline Amitriptilina & & & & & & & \\
\hline Imipramina & & & & & & & \\
\hline Flunarizina & & & & & & & \\
\hline Nimodipino & & & & & & & \\
\hline Propranolol & & & & & & & \\
\hline Metoprolol & & & & & & & \\
\hline Topiramato & & & & & & & \\
\hline Gabapentina & & & & & & & \\
\hline Coenzima $\mathrm{Q}$ & & & & & & & \\
\hline Melatonina & & & & & & & \\
\hline Vitamina B2 & & & & & & & \\
\hline
\end{tabular}

Rojo: no se recomienda. Amarillo: se ha reportado como evento adverso pero en un porcentaje bajo de pacientes. Verde: debe considerarse como una buena alternativa.

Fuente: tomado y modificado de (31-36)

(47). Hoy en día su uso está a criterio del médico tratante y se recomienda considerar el acetaminofén como la primera línea de tratamiento (48).

Los AINES están contraindicados en pacientes con antecedentes de hemorragia gastrointestinal y enfermedad renal. El diclofenaco también debe evitarse en pacientes con antecedente de falla cardiaca o infarto de miocardio (30). Adicionalmente, debe vigilarse el potencial riesgo de transaminitis leve secundario a AINES, ergotaminicos y acetaminofen en pacientes críticamente enfermos.

En pacientes con infección por covid-19 en quienes se esté considerando manejo con ritonavir/lopinavir, se debe evitar el uso de ergotamínicos; ambos medicamentos son inhibidores potentes del CYP3A4, lo que favorece los síndromes ergotamínicos con síntomas vasomotores intensos que conducen a insuficiencia vascular periférica, isquemia en extremidades, coma e incluso la muerte $(49,50)$.

En el caso de triptanes, su uso no se recomienda en pacientes con antecedentes de ataque cerebrovascular (ACV) (29). Esto es importante en el contexto de la actual pandemia, porque los estudios han encontrado una frecuencia de ACV hasta del 2,8\% en pacientes hospitalizados por covid-19 (6). Los triptanes también deben evitarse en compromiso hepático o deterioro renal con una tasa de filtración glomerular menor del $30 \mathrm{ml} / \mathrm{min} / 1,73 \mathrm{~m} 2$ (31). Especial mención debe hacerse al eletriptan, que posee alta afinidad a proteínas y metabolismo hepático a través de la CYP3A4 (51).

La clorpromazina es útil en el manejo agudo de la migraña, sin embargo, su uso puede asociarse a mareo, somnolencia, síntomas extrapiramidales y a prolongación del QT, en especial en combinación con hidroxicloroquina, cloroquina o azitromicina (52).

Los esteroides son una alternativa en las terapias de transición, pero hay controversia en torno a su seguridad en relación con el riesgo teórico de incremento de replicación viral observado en pacientes con SARS-CoV (53). Sin embargo, aún no hay suficiente información al respecto y su uso en pacientes sanos, sin infección por covid-19, queda a discreción del médico tratante.

De acuerdo con los mecanismos de acción y la presencia de enfermedad por covid-19 se debería considerar potencialmente evitar el uso de betabloqueadores, debido al potencial compromiso de la capacidad de respuesta del sistema simpático.

Es importante aclarar que los medicamentos para la terapia específica de covid-19 aún están en investigación, no obstante, su uso clínico en la pandemia se hace la siguiente recomendación, por la interacción que puedan tener con los medicamentos para el manejo a gudo y preventivo de la cefalea (tablas 9-11). 
Tabla 8. Nuevos tratamientos

\begin{tabular}{|c|c|c|c|c|c|}
\hline Medicamento & $\begin{array}{l}\text { Tipo de } \\
\text { molécula }\end{array}$ & $\begin{array}{l}\text { Mecanismo } \\
\text { de acción }\end{array}$ & $\begin{array}{l}\text { Vía de } \\
\text { administración }\end{array}$ & $\begin{array}{l}\text { Frecuencia de } \\
\text { administración }\end{array}$ & $\begin{array}{l}\text { Riesgos } \\
\text { potenciales }\end{array}$ \\
\hline Lasmiditan & & $\begin{array}{l}\text { Agonista receptor } \\
5 \mathrm{HT} 1 \mathrm{~F}\end{array}$ & V.o & Manejo agudo & $\begin{array}{l}\text { Mareo, confusión, } \\
\text { bradicardia, } \\
\text { hipertensión }\end{array}$ \\
\hline Erenumab & MAb IgG2 & $\begin{array}{l}\text { Antagonista receptor } \\
\text { de CGRP }\end{array}$ & s.c & Mensual & $\begin{array}{l}\text { Estreñimiento, } \\
\text { hipertensión arterial, } \\
\text { enfermedad } \\
\text { cardiovascular }\end{array}$ \\
\hline Galcanezumab & MAb IgG4 & Ligando de CGRP & s.c & Mensual & $\begin{array}{l}\text { Enfermedad } \\
\text { cardiovascular, } \\
\text { enfermedad vascular } \\
\text { periférica }\end{array}$ \\
\hline Fremanezumab & MAb IgG2 & Ligando de CGRP & s.c & Trimestral & $\begin{array}{l}\text { Enfermedad } \\
\text { cardiovascular }\end{array}$ \\
\hline Eptinezumab & MAb IgG1 & Ligando de CGRP & i.v & Trimestral & $\begin{array}{l}\text { Enfermedad } \\
\text { cardiovascular }\end{array}$ \\
\hline Ubrogepant & MAb & Antagonista del receptor & v.o & Manejo agudo & $\begin{array}{l}\text { Requiere ajuste de la } \\
\text { dosis en enfermedad } \\
\text { hepática o renal } \\
\text { severa }\end{array}$ \\
\hline Rimegepant & $\mathrm{MAb}$ & $\begin{array}{l}\text { Antagonista del receptor } \\
\text { de CGRP }\end{array}$ & v.o & Manejo agudo & $\begin{array}{l}\text { Suspender en } \\
\text { enfermedad hepática } \\
\text { o renal severa }\end{array}$ \\
\hline
\end{tabular}

IgG: inmunoglobulina, CGRP: péptido relacionado con el gen de la calcitonina, MAb: anticuerpo monoclonal; .v.o: via oral; s.c: subcutánea; i.v: intravenosa.

Fuente: tomado y modificado de (41-44)

\section{REFERENCIAS}

1. Baig AM, Khaleeq A, Ali U, Syeda H. Evidence of the Covid19 virus targeting the CNS: tissue distribution, host-virus interaction, and proposed neurotropic mechanisms. ACS Chem Neurosci. 2020;11(7):995-8.

2. Paniz-Mondolfi A, Bryce C, Grimes Z, Gordon RE, Reidy J, Lednicky J, et al. Central nervous system involvement by severe acute respiratory syndrome Coronavirus -2 (SARS-CoV-2). J Med Virol. 2020 Apr 21. doi: 10.1002/jmv.25915.

3. Moriguchi T, Harii N, Goto J, Harada D, Sugawara H, Takamino J, et al. A first case of meningitis/encephalitis associated with SARS-Coronavirus-2. Int J Infect Dis. 2020;94:55-8.

4. Chen T, Wu D, Chen H, Yan W, Yang D, Chen G, et al. Clinical characteristics of 113 deceased patients with coronavirus disease 2019: retrospective study. BMJ 2020; Mar 26. doi: 10.1136/bmj.m1091.

5. Chang D, Lin M, Wei L, Xie L, Zhu G, Dela Cruz CS, et al. Epidemiologic and clinical characteristics of novel coronavirus infections involving 13 patients outside Wuhan, China. JAMA. 2020;323(11):1092-3.
6. Mao L, Jin H, Wang M, Hu Y, Chen S, He Q, et al. Neurologic manifestations of hospitalized patients with coronavirus disease 2019 in Wuhan, China. JAMA Neurol. 2020 Apr 10. doi: 10.1001/jamaneurol.2020.1127.

7. Huang C, Wang Y, Li X, Ren L, Zhao J, Hu Y, et al. Clinical features of patients infected with 2019 novel coronavirus in Wuhan, China. Lancet. 2020;395(10223):497-506.

8. Chen N, Zhou M, Dong X, Qu J, Gong F, Han Y, et al. Epidemiological and clinical characteristics of 99 cases of 2019 novel coronavirus pneumonia in Wuhan, China: a descriptive study. Lancet. 2020;395(10223):507-13.

9. Tian S, Hu N, Lou J, Chen K, Kang X, Xiang Z, et al. Characteristics of Covid-19 infection in Beijing. J Infect. 2020;80(4):401-6.

10. Xu XW, Wu XX, Jiang XG, Xu KJ, Ying LJ, Ma CL, et al. Clinical findings in a group of patients infected with the 2019 novel coronavirus (SARS-Cov-2) outside of Wuhan, China: Retrospective case series. BMJ. 2020 Feb 19. doi: 10.1136/ bmj.m606.

11. Volcy Gómez M. Cefalea en el servicio de urgencias: nuevos 
Tabla 9. Interacción de medicamentos para el manejo agudo

\begin{tabular}{|c|c|c|c|c|c|}
\hline & Remdesivir & $\begin{array}{l}\text { Hidroxicloroquina } \\
\text { /cloroquina }\end{array}$ & Tocilizumab & Azitromicina & $\begin{array}{l}\text { Lopinavir/ } \\
\text { ritonavir }\end{array}$ \\
\hline Acetaminofén & $\mathrm{A}$ & B & $\mathrm{A}$ & $\mathrm{A}$ & $\mathrm{A}$ \\
\hline $\begin{array}{l}\text { Acido acetil } \\
\text { salicilico }\end{array}$ & $\mathrm{A}$ & $\mathrm{C}$ & $\mathrm{A}$ & $\mathrm{A}$ & $\mathrm{A}$ \\
\hline Diclofenaco & $\mathrm{A}$ & $\bar{A}$ & $\mathrm{~A}$ & $\bar{A}$ & $\mathrm{~A}$ \\
\hline Ibuprofeno & $\mathrm{A}$ & $\mathrm{A}$ & $\mathrm{A}$ & $\mathrm{A}$ & $\mathrm{A}$ \\
\hline Indometacina & $\mathrm{A}$ & $\mathrm{A}$ & $\mathrm{A}$ & $\mathrm{A}$ & $\mathrm{A}$ \\
\hline Ketoprofeno & $\mathrm{A}$ & $\mathrm{A}$ & $\mathrm{A}$ & $\mathrm{A}$ & $\mathrm{A}$ \\
\hline Naproxeno & $\mathrm{A}$ & $\mathrm{A}$ & $\mathrm{A}$ & $\mathrm{A}$ & $\mathrm{A}$ \\
\hline Sumatriptan & $\mathrm{A}$ & $\mathrm{A}$ & $\mathrm{A}$ & $\mathrm{A}$ & $\mathrm{A}$ \\
\hline Eletriptan & $\mathrm{A}$ & $\mathrm{A}$ & $\mathrm{A}$ & $\mathrm{A}$ & $\mathrm{X}$ \\
\hline Zolmitriptan & $\mathrm{A}$ & $\mathrm{A}$ & $\mathrm{A}$ & $\mathrm{A}$ & $\mathrm{A}$ \\
\hline Ergotamina & $\mathrm{A}$ & $\mathrm{A}$ & $\mathrm{A}$ & $\mathrm{A}$ & $\mathrm{X}$ \\
\hline Ketorolaco & $\mathrm{A}$ & $\mathrm{A}$ & $\mathrm{A}$ & $\mathrm{A}$ & $\mathrm{A}$ \\
\hline Celecoxib & $\mathrm{A}$ & $\mathrm{A}$ & $\mathrm{A}$ & $\mathrm{A}$ & $\mathrm{A}$ \\
\hline Eterocoxib & $\mathrm{A}$ & $\mathrm{A}$ & $\mathrm{C}$ & $\mathrm{A}$ & $\mathrm{A}$ \\
\hline
\end{tabular}

Escalas de riesgo: $\mathrm{A}=$ no se conocen interacciones. $\mathrm{B}=$ no requiere cambiarse. $\mathrm{C}=$ vigilancia estrecha. $\mathrm{D}=$ considere modificar la terapia. $\mathrm{X}=$ evite la combinación,

Fuente: tomado y modificado de Lexicomp

conceptos en el diagnóstico, la epidemiología y el tratamiento. Acta Neurol Colomb. 2008;24:118-33.

12. Bolay H, Gül A, Baykan B. Covid-19 is a real headache! Headache J Head Face Pain. 2020 May 15. doi: 10.1111/head.13856.

13. Zhu J, Ji P, Pang J, Zhong Z, Li H, He C, et al. Clinical characteristics of 3,062 Covid-19 patients: a meta-analysis. J Med Virol. 2020 Apr 15. doi: 10.1002/jmv.25884.

14. Vincent M, Wang S. Headache Classification Committee of the International Headache Society (IHS) The International Classification of Headache Disorders, 3rd edition. Cephalalgia. 2018;38(1):1-211.
15. Stovner LJ, Nichols E, Steiner TJ, Abd-Allah F, Abdelalim A, Al-Raddadi RM, et al. Global, regional, and national burden of migraine and tension-type headache, 1990-2016: a systematic analysis for the Global Burden of Disease Study 2016. Lancet Neurol;17(11):954-76.

16. Lipton RB, Dodick D, Sadovsky R, Kolodner K, Endicott J, Hettiarachchi J, et al. A self-administered screener for migraine in primary care: The ID migraine TM validation study. Neurology. 2003;61(3):375-82.

17. Detsky ME, McDonald DR, Baerlocher MO, Tomlinson GA, McCrory DC, Booth CM. Does this patient with headache 
Tabla 10. Interacción de medicamentos para la terapia de transición

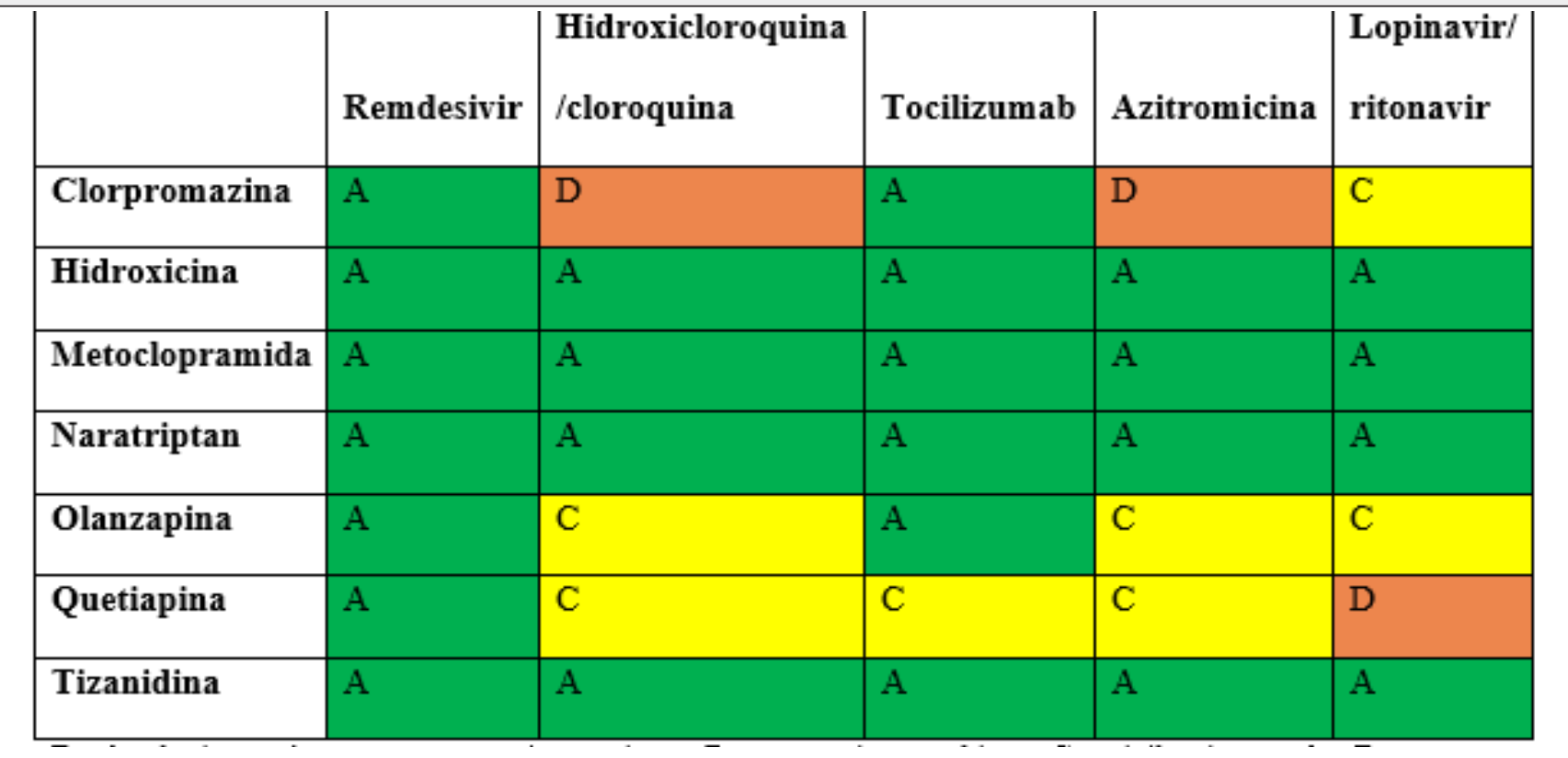

Escalas de riesgo: $\mathrm{A}=$ no se conocen interacciones. $\mathrm{B}=$ no requiere cambiarse. $\mathrm{C}=$ vigilancia estrecha. $\mathrm{D}=$ considere modificar la terapia. $\mathrm{X}=$ evite la combinación

Fuente: tomado y modificado de Lexicomp

have a migraine or need neuroimaging? J Am Med Assoc. 2006;296(10):1274-83.

18. Ezpeleta D, García D. Manual Covid-19 para el neurólogo general. Madrid: Sociedad Española de Neurología; 2020.

19. Katz M. The cost-effective evaluation of uncomplicated headache. Med Clin North Am. 2016;100(5):1009-17.

20. Ong JJY, Bharatendu C, Goh Y, Tang JZY, Sooi KWX, Tan YL, et al. Headaches associated with personal protective equipment - a cross-sectional study among frontline healthcare workers during Covid-19. Headache. 2020;60(5):864-77.

21. Krymchantowski AV. Headaches due to external compression. Curr Pain Headache Rep. 2010;14(4):321-4.

22. Marmura MJ. Triggers, protectors, and predictors in episodic migraine. Curr Pain Headache Rep. 2018;22(12):81. doi: 10.1007/s11916-018-0734-0.

23. Bernstein CA, Nir RR, Noseda R, Fulton AB, Huntington $\mathrm{S}$, Lee AJ, et al. The migraine eye: distinct rod-driven retinal pathways' response to dim light challenges the visual cortex hyperexcitability theory. Pain. 2019;160(3):569-78.

24. Müller KI, Alstadhaug KB, Bekkelund SI. Telemedicine in the management of non-acute headaches: A prospective, openlabelled non-inferiority, randomised clinical trial. Cephalalgia. 2017;37(9):855-63.

25. Qubty W, Patniyot I, Gelfand A. Telemedicine in a pediatric headache clinic: A prospective survey. Neurology. 2018;90(19):e1702-5.

26. Müller KI, Alstadhaug KB, Bekkelund SI. A randomized trial of telemedicine efficacy and safety for nonacute headaches. Neurology. 2017;89(2):153-62.
27. Grazzi L, Rizzoli P. The adaptation of management of chronic migraine patients with medication overuse to the suspension of treatment protocols during the Covid-19 pandemic: lessons from a tertiary headache center in Milan, Italy. Headache. 2020 Apr 30. doi: 10.1111/head.13825.

28. Uribe B, Takeuchi Y, Miranda G. Consenso de expertos de la Asociación Colombiana de Neurología para el tratamiento preventivo y agudo de la migraña Acta Neurol Colomb. 2014;30(3):175-85.

29. Jamieson DG. The safety of triptans in the treatment of patients with migraine. Am J Med. 2002;112(2):135-40.

30. Szperka CL, Ailani J, Barmherzig R, Klein BC, Minen MT, Halker Singh RB, et al. Migraine care in the era of Covid-19: clinical pearls and plea to insurers. Headache. 2020;60(5):833-42.

31. Brunton L, Chabner B, Knollmann B. Goodman \& Gilman: las bases farmacológicas de la terapéutic, 13 edición. AccessMedicina, McGraw-Hill Medical Pub; 2018.

32. Silberstein SD. Preventive migraine treatment. Continuum (Minneap Minn) 2015;21(4):973-89.

33. Schwedt TJ. Preventive therapy of migraine. Contin Lifelong Learn Neurol. 2018;24(4):1052-65.

34. Thompson DF, Saluja HS. Prophylaxis of migraine headaches with riboflavin: A systematic review. J Clin Pharm Ther. 2017;42(4):394-403.

35. Foley HM, Steel AE. Adverse events associated with oral administration of melatonin: A critical systematic review of clinical evidence. Complement Ther. 2019;42:65-81.

36. Hidaka T, Fujii K, Funahashi I, Fukutomi N, Hosoe K. Safety assessment of coenzyme Q10 (CoQ10). BioFactors. 2008;32(14):199-208. 
Tabla 11. Interacción de medicamentos para el manejo preventivo

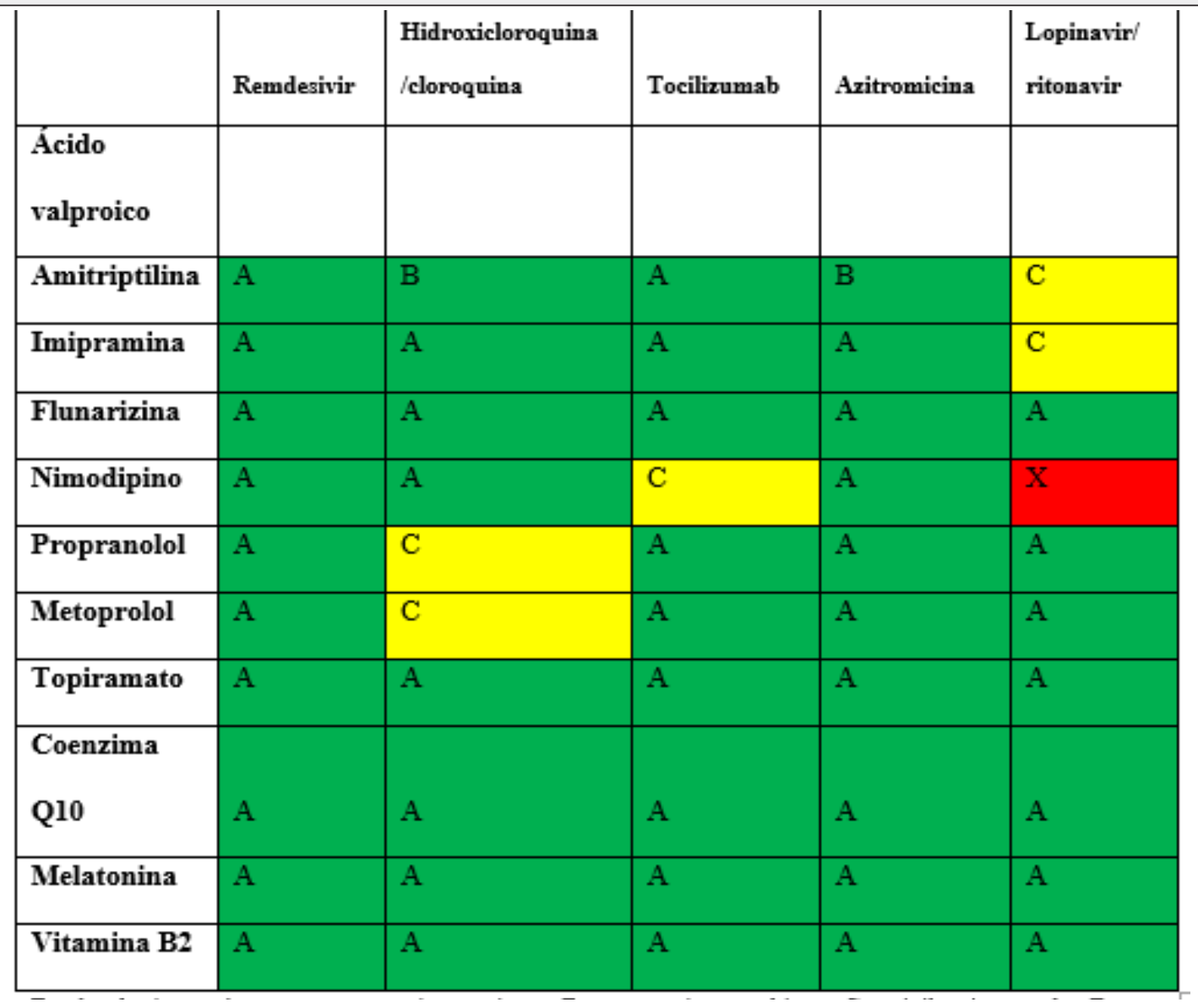

Escalas de riesgo: $\mathrm{A}=$ no se conocen interacciones. $\mathrm{B}=$ no requiere cambiarse. $\mathrm{C}=$ vigilancia estrecha. $\mathrm{D}=$ considere modificar la terapia. $\mathrm{X}=$ evite la combinación

Fuente: tomado y modificado de Lexicomp

37. Ali A. Delay in onabotulinumtoxinA treatment during the Covid-19 pandemic-perspectives from a virus hotspot. Headache. 2020;60(6):1183-6.

38. Ministerio de Salud de Colombia. Orientaciones para la restauración gradual de los servicios de salud en las fases de mitigación y control de la emergencia sanitaria por Covid-19 en Colombia. 2020 [citado 2020 mayo 19]. Disponible en: https:/ / www.minsalud.gov.co/Ministerio/Institucional/Procesos y procedimientos/PSSS05.pdf

39. Ministerio de Salud de Colombia. Lineamientos para prevención control y reporte de accidente por exposición ocupacional al covid-19 en instituciones de salud. 2020 [citado 2020 mayo 19]. Disponible en: https:/ /www.minsalud.gov.co/Ministerio/ Institucional/Procesos y procedimientos/GPSG04.pdf

40. ClinicalTrials.gov. Safety and efficacy trial of vazegepant intranasal for hospitalized patients with covid-19 requiring supplemental oxygen [full text view].

41. MaassenVanDenBrink A, Meijer J, Villalón CM, Ferrari MD. Wiping out CGRP: potential cardiovascular risks. Trends Pharmacol Sci. 2016;37(9):779-88.
42. Kee Z, Kodji X, Brain SD. The role of calcitonin gene related peptide (CGRP) in neurogenic vasodilation and its cardioprotective effects. Front Physiol. 2018 Sep 19; 9:1249. doi: 10.3389/fphys.2018.01249.

43. Borkum JM. CGRP and brain functioning: cautions for migraine treatment. Headache. 2019;59(8):1339-57.

44. Scuteri D, Adornetto A, Rombolà L, Naturale MD, Morrone LA, Bagetta G, et al. New trends in migraine pharmacology: Targeting calcitonin gene-related peptide (CGRP) with monoclonal antibodies. Front Pharmacol. 2019;9;10:363. doi: 10.3389/fphar.2019.00363

45. Fang L, Karakiulakis G, Roth M. Are patients with hypertension and diabetes mellitus at increased risk for Covid-19 infection? Lancet Respir Med. 2020;8(4):e21.

46. FDA advises patients on use of non-steroidal anti-inflammatory drugs (NSAIDs) for Covid-19 [citado 2020 mayo 12]. Disponible en: https://www.fda.gov/drugs/drug-safetyand-availability/fda-advises-patients-use-non-steroidal-antiinflammatory-drugs-nsaids-covid-19

47. The use of non-steroidal anti-inflammatory drugs (NSAIDs) in 
patients with Covid-19 [citado 2020 mayo 12]. Disponible en: https://www.who.int/publications-detail/the-use-of-non-steroidal-anti-inflammatory-drugs-(nsaids)-in-patients-with-covid-19

48. MaassenVanDenBrink A, de Vries T, Danser AHJ. Headache medication and the Covid-19 pandemic. J Headache Pain. 2020; 21(1):38. doi: 10.1186/s10194-020-01106-5.

49. Spiegel M, Schmidauer C, Kampfl A, Sarcletti M, Poewe W. Cerebral ergotism under treatment with ergotamine and ritonavir. Neurology. 2001;57(4):743-4.

50. Acle S, Roca F, Vacarezza M, Rocha AÁ. Ergotismo secundario a la asociación ergotamina-ritonavir. A propósito de 3 casos. Rev Med Chil. 2011;139(12):1597-600.
51. Capi M, Curto M, Lionetto L, De Andrés F, Gentile G, Negro A, et al. Eletriptan in the management of acute migraine: An update on the evidence for efficacy, safety, and consistent response. Ther Adv Neurol Disord. 2016;9(5):414-23.

52. Meid AD, Bighelli I, Mächler S, Mikus G, Carrà G, Castellazzi $\mathrm{M}$, et al. Combinations of QTc-prolonging drugs: towards disentangling pharmacokinetic and pharmacodynamic effects in their potentially additive nature. Ther Adv Psychopharmacol. 2017;7(12):251-64.

53. Russell B, Moss C, Rigg A, Van Hemelrijck M. Covid-19 and treatment with NSAIDs and corticosteroids: should we be limiting their use in the clinical setting? Ecancermedicalscience. 2020 Mar 30; 14:1023. doi: 10.3332/ecancer.2020.1023. 